\title{
A Study of WSN and Analysis of Packet Drop During Transmission
}

\author{
Nagma Shakeel $^{1}$, Mohd Haroon ${ }^{2}$, and Faiyaz Ahmad ${ }^{3}$ \\ ${ }^{1}$ M.Tech Research Scholar, Department of Computer Science \& Engineering, Integral University, Lucknow, India \\ ${ }^{2}$ Associate Professor, Department of Computer Science \& Engineering, Integral University, Lucknow, India \\ ${ }^{3}$ Assistant Professor, Department of Computer Science \& Engineering, Integral University, Lucknow, India
}

Correspondence should be addressed to Nagma Shakeel; nagmashakil5@gmail.com

Copyright (C) 2021 Made Nagma Shakeel et al. This is an open access article distributed under the Creative Commons Attribution License, which permits unrestricted use, distribution, and reproduction in any medium, provided the original work is properly cited.

\begin{abstract}
WSN is a low-power system and are often used in numerous monitoring uses, such as healthcare, environmental, and systemic health surveillance, in addition to military surveillance. It is important to reduce network resource usage since many of these applications need to be installed in locations that are virtually inaccessible to humans. Many protocols for WSN to extend the presence of the network have been established to solve this problem. In the energy efficiency of WSN networks, routing protocols play an important role since they help minimize power usage and response time and provide sensor networks with high data density and service quality. This study also employed a Hopfield neural network and the findings from this study are presented next to each other to enable comparison. This paper also discusses how to easily and accurately capture and handle WSN collisions. Future experiments that require the usage of neural networks and so many fuzzy structures will be able to prevent a crash in these respects.
\end{abstract}

KEYWORDS- WSN-wireless sensor networks, PDT-packet delivery ratio, TP-throughput, e2e-delay

\section{INTRODUCTION}

Information is known as process data, information means some meaningful content, in wireless sensor computing, the information will propagate from one node to another through communication media and the various application protocol [1]. One characteristic of WSN is the ability to communicate through the wireless communication channel and sensor nodes in the real world for detecting and manipulating the specific entity. Any or more of these nodes must work together to achieve their goals. the nodes are linked with oneto-one and one-one employing wireless connection an underpowered wireless sensor network (WSN) can connect and function together to achieve a shared objective. They can function in highly dynamic settings, such as combat and surveillance environments. Since they operate on their own, WSNs are seldom unattended, several novel attacks are feasible. Recently, owing to their large acceptance in both military and civilian contexts, WSNs have gained a lot of coverage. WSN are widely used in covert and often adversarial locations such as the military and the domestic intelligence services. Thus, authentication protocols that accomplish the overall goals of congeniality, data privacy, nonrepudiation, and trustworthiness are critical to maintaining the integrity of the network. These days, the world's technology is going to base on the artificial intelligence technique, several artificial intelligence devices and protocols are used for various purposes.in wireless sensor nodes, artificial intelligence agents and protocols are playing a significant role [2].

\section{A. Sensor nodes}

The sensor nodes would be on the network handling the tasks. Although Task Manager measurements and queries could be taking place, the sensor nodes may send data based on these algorithms. Computations can be made on a node, depending on the device requirements If the model has been built, it may either transfer the data to the other nodes or give it to the Task Manager as is. In sensor nodes, the sensor may be either a source or a relay: The root is a means of figuring out and obtaining what is wanted. The world then is the source. Meanwhile, a sink or actuator is a device that is involved in receiving data from a sensor.

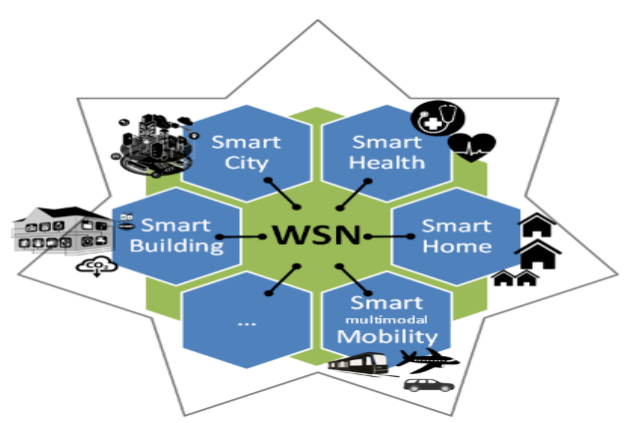

Fig. 1: Illustration of sensor network and peripherals [15] 


\section{B. System Components and Operations in a Wireless Sensor Network}

\section{- Communication Architecture}

This section focuses on the sensor infrastructure. The sensors in the nodes' components and sub-of-world will be tested. We begin with a brief architectural and data-processing overview of the wireless sensor network before moving on to the protocols and systems that are employed in building a sensor network. to consider the total amount of power and hardware/software savings is crucial One of the aims of this study is to collect data on sensor nodes and to suggest hardware for their usage. More specific guidance may be obtained by referring to the equipment in depth.

\section{- Sensor Node in communication architecture}

In the first instance, a sensor is a low-power wireless device that is used in communication. A sensor node will usually act as a data processor, data repository, and communicator all at the same time. The standard sensor node contains the controller, memory, sensors, communication system, and power supply in this list (see Figure 2). The task of a controller is to perform is to process all the pertinent data, able to implement arbitrary algorithms. Data and program memory are the functions in memory. Input sensors and output actuators are the methods of communication with the environment. Any or all of these devices enable one to monitor or regulate environmental parameters. The unit interacts using radio waves. Lastly, the power supply is required to sustain the electronic components. One of the maximum significant architecture factors in WSN is power efficiency Thus, these entangled elements need to make the most of the minimum number of resources with the will to work.

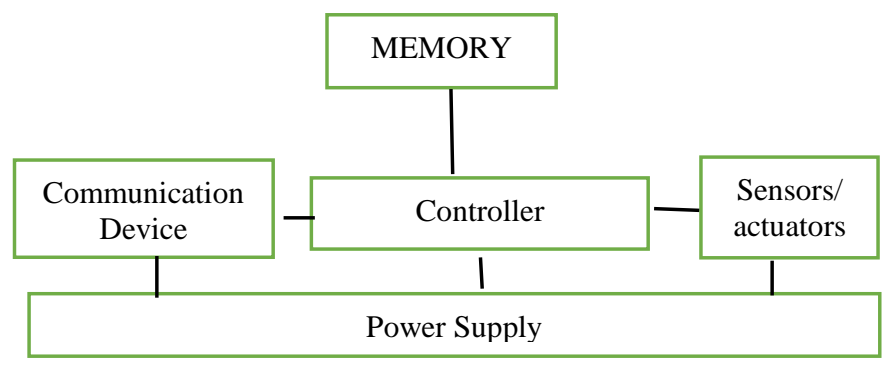

Fig. 2: Overview of sensor node hardware component. [14]

\section{BACKGROUND}

Each responsive node senses an occurrence in a densely distributed sensor network and broadcasts it via multi-hop connexions to a particular party. additional data transmission and control packets are needed for sensor nodes installed near the basin node and are therefore subject to much higher energy depletion rates and therefore have much less expected network existence. in the paper, in the presence of the issue of power holes in the wireless sensor network, they addressed the performance of ad-hoc on-demand distance vector, dynamic state routing, and Temporally Ordered Routing Algorithm protocols. for each protocol, productivity, average power usage, and end-to-end latency, work performance, packet transmission rate, packet drop rate, active nodes, and routing expenses under various node densities were demonstrated. to mitigate this problem, the effectiveness of some of the prevalent approaches was applied and simulation conclusions were used to authenticate the study. compared with ad-hoc on-demand distance vector and Temporally Ordered Routing Algorithm, the number of active nodes in the dynamic state routing protocol is larger [3]. In recent years, because of the heavy burden of transmitting data to sensor nodes near the basin, WSN has faced the overall energy issue as the most significant problem. The Mobile Sink (MS) method is the best-proven alternative defined by the new existing approaches to the entire energy crisis. Allowing MS to visit each node to collect data, though, results in high data latency, which might not be possible in applications that are lag-sensitive. In this article, restricted mobile pelvic activity is also regarded, since MS ends at an imperfect number of sites listed as residence sites and all nodes publish their data at neighboring residence sites. Therefore, the suggested protocol range parameter is used in this article to preserve network coverage even though certain nodes are down. The suggested routing algorithm, focused on the residency positions, guarantees that all data in the cluster is propagated to the MS following the minimum hop route to minimize the gap in the transmission of data. About dissimilar constraints such as network life, coverage rate, energy efficiency, packet transmission rate, end-to-end delay, etc., the experimental findings reveal the efficacy of the proposed protocol over several modern protocols [4]. The growing need for different facets of the usage of wireless sensor applications renders service quality one of the maximum critical problems of wireless sensor applications. It is very difficult to maintain the standard of operation in wireless sensor (WSN) since the tools accessible to the numerous sensors and applications running on those networks have multiple limitations in terms of design and specifications. QoS has historically concentrated on the level of the network, paying attention to metrics like latency, efficiency, and volatility. The decentralized and complex topology of WSN has several essential criteria, including reducing power usage and extending the network's existence. The simulation results include overall network output that, along with a variety of dead nodes, total power consumption, community header settings, and performance, depends primarily on some factors [5]. There are several nodes in WSN whose primary functions are to track and manage ecosystems. Additionally, depending on network use, the sensor nodes are spread. The power usage of the sensor nodes one of the principal problems of this type of network. Nodes close to the basin serve as a data transfer interface in fixed basin networks for other nodes to sink. Also, the pelvic motion orientation route for the transmission of $\mathrm{CH}$ data is observed by the Hopfield neural network. Also, detailed simulations conducted in an NS-2 setting can test the WSNFAHN technology here. Compared to modern schemes focused on efficiency measurements such as packet 
distribution rate (PDR), average throughput, detection rate, and life, the supremacy of the WSN-FAHN technique is shown by simulation outcomes. Useful network when the average residual capacity is reduced [6]. High saturation streams increase the capacity for collision and congestion in data transmission in the field of Wireless Multimedia Sensor Networks (WMSN), which dramatically degrades QoS efficiency. To ensure the consistency of operation, multichannel deployment technology is also extended to simultaneous transmission. Find an efficient performance measure and design an efficient performance selection or scoring method centered on the required parameters that can be used for the multichannel allocation process as parameter inputs. The findings reveal that, after some phases of the selflearning phase, the deep reinforcement learning paradigm effectively achieves a stronger multichannel allocation approach. Due to the discovery of the main atmosphere in the first step of the learning phase, the primary weight fusion system helps one to minimize energy expenditure [7]. WSN has tremendous potential to serve numerous applications such as military and intelligent transport, wellbeing and medical sectors, environmental surveillance, etc. This paper outlined and examined the root and description of wireless sensor network faults focused on the national and international study status of fault diagnostic technologies. Centralized techniques and distributed algorithms were then developed according to the main location of the diagnostic error in error detection techniques. Finally, there is talk of potential research and development problems for sensor network malfunctions [8].

A very promising wireless technology approach for industrial applications is WSN(WSN). However, four key characteristics, namely, energy consumption, scalability, flexibility, and timeliness, must be met for the reliable implementation of WSN networks in an industrial setting. However, this increase in communication efficiency is accomplished at the cost of higher latency, which might not be sufficient for strict timing specifications in industrial applications. Often, this is only feasible in certain circumstances by selecting MAC parameter values that are not officially allowed by the specification [9]. In missioncritical industrial applications and delay critical industrial applications, WSN(WSN) is the most common because they have low latency and efficient message delivery. The stable relationship between the sump and the sensor nodes is very necessary for applications including gas leak detection, pressure management, and industrial process control, etc. Sensor nodes are positioned very densely in WSN in diverse locations and also without a particular network configuration. The positioning of the sensor nodes plays an enormous role in an industrial setting and, most significantly, improves overall machine productivity by accurately communicating optimized readings and ensuring optimum protection for industrial equipment. By positioning the sensor nodes in three separate topological designs: linear, level one, and split level one, we measured the efficiency of the wireless sensor network (WSN) [10]. In the era of networked networking, WSN plays a crucial function. With a vast number of sensors connecting within the range of the network, exponential development in connectivity technologies has allowed the wireless sensor network to evolve faster and faster. This present several strategies for soft computing and data mining that will be applied to evaluate the answer to these issues in the wireless sensor network. These strategies can function quickly and reliably and cause issues with the wireless sensor network [11].

Table 1: Extracted research previews

\begin{tabular}{|c|c|c|c|}
\hline Authors & $\begin{array}{l}\text { Technique } \\
\text { Used }\end{array}$ & $\begin{array}{l}\text { Area of } \\
\text { research }\end{array}$ & Result \\
\hline $\begin{array}{l}\text { Sharma } \\
\& \\
\text { Lobiyal } \\
(2015)\end{array}$ & $\begin{array}{l}\text { ad-hoc on- } \\
\text { demand } \\
\text { distance vector, } \\
\text { dynamic state } \\
\text { routing and } \\
\text { temporally } \\
\text { ordered routing } \\
\text { algorithm }\end{array}$ & WSN & $\begin{array}{lr}\text { performance of ad- } \\
\text { hoc on-demand } \\
\text { distance rector, } \\
\text { dynamic state routing } \\
\text { and temporally } \\
\text { ordered routing } \\
\text { algorithm protocols }\end{array}$ \\
\hline $\begin{array}{l}\text { Roy et. } \\
\text { al. } \\
(2020)\end{array}$ & $\begin{array}{l}\text { block-based } \\
\text { routing } \\
\text { protocol }\end{array}$ & WSN & $\begin{array}{l}\text { suggested routing } \\
\text { algorithm, focused on } \\
\text { the residency } \\
\text { positions, guarantees } \\
\text { that all data in the } \\
\text { cluster is propagated } \\
\text { to the ms following } \\
\text { the minimum hop } \\
\text { route to minimize the } \\
\text { gap in the broadcast } \\
\text { of data }\end{array}$ \\
\hline $\begin{array}{l}\text { rai et al. } \\
(2017)\end{array}$ & $\begin{array}{l}\text { quality of } \\
\text { facilities (Qos) } \\
\text { for wireless } \\
\text { sensor network }\end{array}$ & WSN & $\begin{array}{l}\text { simulation results } \\
\text { include overall } \\
\text { network output that, } \\
\text { along with a variety of } \\
\text { dead nodes, total } \\
\text { power consumption, } \\
\text { community header } \\
\text { settings, and } \\
\text { performance, depends } \\
\text { primarily on a number } \\
\text { of factors }\end{array}$ \\
\hline $\begin{array}{l}\text { Fotohi \& } \\
\text { Bari } \\
(2020) .\end{array}$ & $\begin{array}{l}\text { filtering } \\
\text { algorithm } \\
\text { based on } \\
\text { firefly, and the } \\
\text { neural network } \\
\text { of Hopfield } \\
\text { (WSN-fahn) }\end{array}$ & WSN & $\begin{array}{l}\text { to modern schemes } \\
\text { focused on efficiency } \\
\text { measurements such as } \\
\text { packet distribution } \\
\text { rate (pdr), average } \\
\text { throughput, detection } \\
\text { rate, and life, the } \\
\text { supremacy of the } \\
\text { wsn-fahn technique is } \\
\text { shown by simulation } \\
\text { outcomes }\end{array}$ \\
\hline $\begin{array}{l}\text { Liu } \\
(2017)\end{array}$ & $\begin{array}{l}\text { utilizing the } \\
\text { deep q network } \\
\text { augmented } \\
\text { learning system } \\
\text { 9DQMC), }\end{array}$ & $\begin{array}{l}\text { wireless } \\
\text { multimedia } \\
\text { sensor } \\
\text { networks } \\
\text { (WMSN) }\end{array}$ & $\begin{array}{l}\text { present the } \\
\text { incorporation of a } \\
\text { progressive machine } \\
\text { learning methodology } \\
\text { into the multichannel } \\
\text { personalization } \\
\text { process }\end{array}$ \\
\hline
\end{tabular}




\section{PROBLEM STATEMENT}

It is quite hard to detect the suspicious activities in WSN communication. This research has main focus of detection of Malicious Nodes in WSN using Neural Network. For this we are considering the Neural Networks as the mechanism to perform the random creation of test and differentiate the time domain activities. The performance of every classifier is check based on various parameters like packet delivery, endto-end transmission, and delay, and throughput.

\section{METHODOLOGY}

The first phase in understanding the efficiency of wireless communications in the ecosystems and densities in which we anticipate sensor networks to be implemented is taken in this paper [1,4]. Packet transmission efficiency is the key feature of wireless performance that we are worried about. The key output metric is, to be more accurate, the packet loss rate (the number of packets sent but not returned within the time frame) or the supplementary receipt rate. In a wireless communication device, several, many variables control the efficiency of packet representation: environment, network topology, traffic trends, and therefore the real physical phenomena that drive the communication operation of the node. It is hard to distinguish these phenomena to research the effect of multiple influences on the efficiency of beam transmission. Also, from a more mechanical viewpoint, this white paper addresses the packet transmitting performance of two different layers in the network stack: the physical layer and the layer of medium access. We do this in a structured manner, which suggests that we have a certain influence over the topology of the network, the output of traffic, and the time and length of our experiments $[5,6]$. Our studies are not fully monitored, however, since our measures are sensitive to external influences, such as environmental variability. This is deliberate because we want (at least in part) to consider how contact is influenced by environmental influences. We would use the widely utilized model of the sensor network since it helps to explain the distribution of the data packet on the sensor network. Implemented Network Stack in Mica mote [7], RF Monolithic [8], Tiny OS [9]. This is a shifting target; the numerous radios and protocols will start to move as the platform begins to develop. We fix this by not creating a single difference that can be overridden by the current platform's gradual progress and by mentioning our assumptions that are likely to be influenced by technical shifts. We discuss these experiences in more detail in the following subsections.

\section{A. Proposed Algorithm}

Create a network of 20 nodes organized in elliptical form.

Select the source and destination of the node and the node of the sensor.

It's though (data is not received by destination)

Reflect this

If so (sensor node detect collision)

And,
Apply a neural pattern recognition network to alter the location of the node where the collision is observed.

And launch the transmission again from the source node.

Now, Else

Transmit the data from one node to the next.

End if it is

End as Network needed.

Exit, exit.

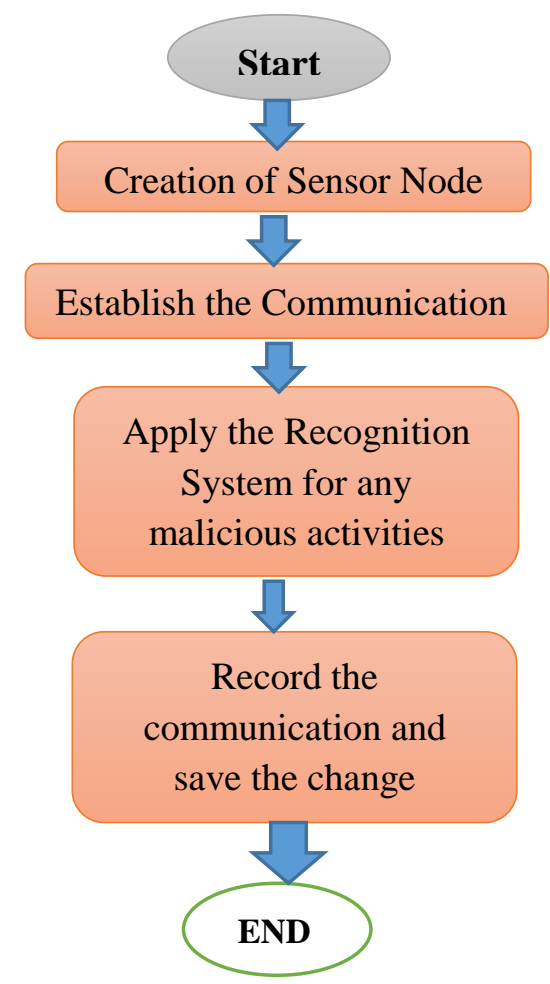

Fig. 2: Flowchart for the proposed algorithm

\section{B. Result Estimator}

The performance of every classifier is check based on various parameters like packet delivery, end-to-end transmission, and delay, and throughput gets. for better comparison for the classifier by the given matrices.

\section{Packet delivery ratio-}

Tt signifies that the total number of messages received by the computing nodes and the total number of messages generated by the computing nodes.

Packet delivery ratio calculated by the given formula

$\mathrm{PDR}=(($ Packet Transmitted- loss packet $) /$ Packet Transmitted) $* 100$.

\section{End to end delay}

Delay means after the successful broadcast of the packet from the source node, packet reach to the destination nodes, then how much time it will take to reach to the destination node that is known as an end-to-end delay.

\section{E. Throughput}

Throughput means the number of data packets set from transmitter end known as throughput. In terms of the 
communication channel. How many packets are traverse to the channel per unit time.

Throughput=total number of packet/ ends to end delay.

These parameters estimated through the pattern recognition method which has to be used under the neural network implement in MATLAB for execution. These parameters reflect the overall communication and any type of malicious activities in WSN.

\section{CONCLUSION AND FUTURE WORK}

A fundamental function of all sensor network implementations is being able to detect intrusion. Sensors networks cannot be used for traditional network defense because of the special features of sensor networks. To begin with, sensors are responsive to production costs since they need a large number of sensors said that sensor networks require a low production cost. As a result, sensor nodes are mostly power, memory, and computation/ Sensor nodes usually draw power from batteries and must be done infrequently. Consideration of network energy use becomes essential for most sensor network protocols. Secondly, Sensor nodes are vulnerable to physical assaults by adversaries who may be put in public locations. Enemies are usually expecting that this research may access a sensor node without leaving any evidence of their activity. Furthermore, the size of sensor networks is immense, and the topology is modified dynamically when certain nodes become unavailable due to lack of resources or become inaccessible due to malfunction.

\section{CONFLICTS OF INTEREST}

The authors declare that they have no conflicts of interest.

\section{REFERENCES}

[1] S. Srivastava, M. Haroon, and A. Bajaj, "Web document information extraction using class attribute approach," Proc. 4th IEEE Int. Conf. Comput. Commun. Technol. ICCCT 2013, pp. 17-22, 2013, doi: 10.1109/ICCCT.2013.6749596.

[2] H. S. Kharkwal, "Automated Task Allotment in Unmanned Submarines by Smart Searching Algorithm," vol. 13, no. 2, 2017.

[3] R. Sharma and D. K. Lobiyal, "Proficiency Analysis of AODV, DSR and TORA Ad-hoc Routing Protocols for Energy Holes Problem in Wireless Sensor Networks," Procedia - Procedia Comput. Sci., vol. 57, pp. 1057-1066, 2015, doi: 10.1016/j.procs.2015.07.380.

[4] M. S. Husain and D. M. Haroon, "an Enriched Information Security Framework From Various Attacks in the Iot," Int. J. Innov. Res. Comput. Sci. Technol., vol. 8, no. 4, 2020, doi: 10.21276/ijircst.2020.8.4.3.

[5] Mazumdar, N., \& Pamula, R. (2020). An energy and coverage sensitive approach to hierarchical data collection for mobile sink based wireless sensor networks. Journal of Ambient Intelligence and Humanized Computing, 1-25.

[6] R. Fotohi, "A Novel Countermeasure Technique to Protect WSN against Denial-of- Sleep Attacks Using Firefly and Hopfield Neural Networks (HNN ) Algorithms."

[7] Liu, Z. (2017). Self-Adaptive Bandwidth Control for Balanced QoS and Energy Aware Optimization in Wireless Sensor
Network (Doc Temporally Ordered Routing Algorithm dissertation).

[8] F. Zhu and J. Wei, "An energy-efficient unequal clustering routing protocol for wireless sensor networks," vol. 15, no. 9, 2019, doi: 10.1177/1550147719879384.

[9] Anastasi, G., Conti, M., \& Di Francesco, M. (2010). A comprehensive analysis of the MAC unreliability problem in IEEE 802.15. 4 wireless sensor networks. IEEE Transactions on Industrial Informatics, 7(1), 52-65.

[10] Rai, P. K., Bharti, P. K., \& Yadav, R. K. (2017). An Approach to Comprehend the Quality of Services (QoS) For Wireless Sensor Network. International Refereed Journal of Reviews and Research, 5(6), 1-5.

[11] M. S. Husain and D. M. Haroon, "an Enriched Information Security Framework from Various Attacks in the Iot," Int. J. Innov. Res. Comput. Sci. Technol., vol. 8, no. 4, 2020, doi: 10.21276/ijircst.2020.8.4.3.

[12] Khan, M. F., Felemban, E. A., Qaisar, S., \& Ali, S. (2013, December). Performance analysis on packet delivery ratio and end-to-end delay of different network topologies in WSN(WSNs). In 2013 IEEE 9th International Conference on Mobile Ad-hoc and Sensor Networks (pp. 324-329). IEEE.

[13] Sunitha, R., \& Chandrika, J. (2016, February). A study on detecting and resolving major issues in wireless sensor network by using data mining and soft computing techniques. In 2016 International Conference on Emerging Trends in Engineering, Technology and Science (ICETETS) (pp. 1-6). IEEE.

[14] Kodali, Ravi \& Muraleedhar, Anupama. (2015). WSN in spice cultivation. 10.1109/ICGCIoT.2015.7380640.

[15] https://www.openaccessgovernment.org/dependable-securetrustable-wireless-sensor-networks/27971/

\section{ABOUT THE AUTHORS}

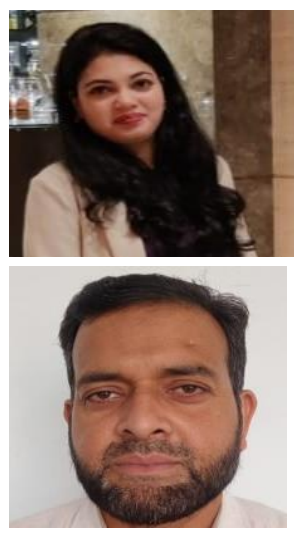

Nagma Shakeel, M.Tech, Research Scholar, Department of Computer Science \& Engineering at Integral University, Lucknow (U.P), India

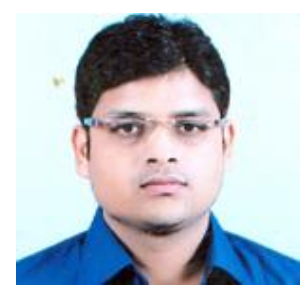

Mohd. Haroon is working as Associate Professor in the Department of Computer Science \& Engineering at Integral University, Lucknow (U.P), India. He has completed his $\mathrm{PhD}$ in "Dynamic Load Balancing in Distribution System" in 2016. research area: distributed machine learning, artificial intelligence.

Faiyaz Ahmad is working as Assistant Professor in the Department of Computer Science \& Engineering at Integral University, Lucknow (U.P), India. He has completed his $\mathrm{PhD}$ in "Soft Computing". research area: Soft Computing machine learning, artificial intelligence, Cyber Security. 